\title{
MiR-375 frequently downregulated in gastric cancer inhibits cell proliferation by targeting JAK2
}

Ling Ding ${ }^{1, *}$, Yanjun $\mathrm{Xu}^{1, *}$, Wei Zhang ${ }^{1}$, Yujie Deng ${ }^{1}$, Misi $\mathrm{Si}^{2}$, Ying $\mathrm{Du}^{3}$, Haomi Yao ${ }^{3}$, Xuyan Liu ${ }^{1}$, Yuehai $\mathrm{Ke}^{1}$, Jianmin $\mathrm{Si}^{3}$, Tianhua Zhou ${ }^{1}$

${ }^{I}$ The Center for Diseases Modeling and Program in Molecular and Cell Biology, Zhejiang University School of Medicine, Hangzhou 310058, China; ${ }^{2}$ Zhejiang University School of Medicine, Hangzhou 310058, China; ${ }^{3}$ Sir Run Run Shaw Hospital, Zhejiang University School of Medicine, Hangzhou 310016, China

Emerging evidence has shown the association of aberrantly expressed microRNAs (miRNAs) with tumor development and progression. However, little is known about the potential role of miRNAs in gastric carcinogenesis. Here, we performed miRNA microarray to screen miRNAs differentially expressed in the paired gastric cancer and their adjacent nontumor tissues and found that miR-375 was greatly downregulated in gastric cancer tissues. Quantitative real-time PCR analysis verified that miR-375 expression was significantly decreased in more than $90 \%$ of primary gastric cancers compared with their nontumor counterparts from patients undergoing gastric resection. Overexpression of miR-375 significantly inhibited gastric cancer cell proliferation in vitro and in vivo. Forced expression of miR-375 in gastric cancer cells significantly reduced the protein level of Janus kinase 2 (JAK2) and repressed the activity of a luciferase reporter carrying the 3'-untranslated region of JAK2, which was abolished by mutation of the predicted miR-375-binding site, indicating that JAK2 may be a miR-375 target gene. Either inhibition of JAK2 activity by AG490 or silencing of JAK2 by RNAi suppressed gastric cancer cell proliferation resembling that of miR-375 overexpression. Moreover, ectopic expression of JAK2 can partially reverse the inhibition of cell proliferation caused by miR-375. Finally, we found a significant inverse correlation between miR-375 expression and JAK2 protein level in gastric cancer. Thus, these data suggest that miR-375 may function as a tumor suppressor to regulate gastric cancer cell proliferation potentially by targeting the JAK2 oncogene, implicating a role of miR-375 in the pathogenesis of gastric cancer.

Keywords: miR-375; JAK2; gastric cancer; proliferation

Cell Research (2010) 20:784-793. doi:10.1038/cr.2010.79; published online 15 June 2010

\section{Introduction}

MicroRNAs (miRNAs) are a new class of naturally occurring small noncoding RNAs that regulate genes

\footnotetext{
*These two authors contributed equally to this work. Correspondence: Jianmin $\mathrm{Si}^{\mathrm{a}}$, Tianhua Zhou ${ }^{\mathrm{b}}$

${ }^{\mathrm{a}}$ Tel: +86-571-8600 6788; Fax: +86-571-8600 6788

E-mail: jianmin_si@zju.edu.cn

${ }^{\mathrm{b}} \mathrm{Tel}:+86-571-8820$ 8258; Fax: +86-571-8820 8070

E-mail: tzhou@zju.edu.cn

Abbreviations: miRNA (microRNA); JAK2 (Janus kinase 2); 3'-UTR (3'-untranslated region); qRT-PCR (quantitative real-time RT-PCR); STAT (signal transducer and activator of transcription)

Received 9 February 2010; revised 16 March 2010; accepted 17 March 2010; published online 15 June 2010
}

by triggering either mRNA degradation or translational repression through either perfect or imperfect binding to the $3^{\prime}$-untranslated region (3'-UTR) of target mRNA [1$3]$. Over the past several years, it has become clear that miRNAs contribute to most, if not all, basic biological processes, such as development, differentiation, apoptosis and cell proliferation [4]. An increasing number of studies have demonstrated that miRNAs can function as potential oncogenes or tumor suppressor genes during the initiation and progression of cancer [4]. It has been estimated that $\sim 50 \%$ of miRNAs are located at or close to fragile sites of regions known to be amplified or deleted in human cancer [5]. Aberrant expression of miRNAs or mutations of miRNA genes have been well described in many types of tumors, including leukemia, lymphoma, breast, lung, pancreas, colon and liver cancers [6]. 
However, the role of miRNAs in gastric cancer remains largely unknown.

Gastric cancer ranks as the fourth most common cancer behind lung, breast and colorectal cancer, and the second leading cause of cancer-related death worldwide $[7,8]$. Like other cancers, the development of gastric cancer is a multistep process with accumulation of genetic and epigenetic changes $[9,10]$. Although a number of miRNAs that are associated with gastric cancer have been identified to date, the role of many of them in tumorigenesis and the underlying mechanism remain to be determined. Recently, we and others have identified some miRNAs deregulated in gastric cancer, such as downregulation of miR-141, miR-143, miR-145, miR-9 and miR-218 [11-14], and upregulation of miR-21, miR150 and 106a [15-18]. Nevertheless, until very recently, the function and regulation of these miRNAs in gastric cancer have hardly been explored.

Here, we provide evidence that miR-375 is one of the most frequently down-regulated miRNAs in gastric cancer tissues obtained from both gastric resection and gastroscopy. MiR-375 was able to significantly inhibit gastric cancer cell proliferation in vitro and in vivo and target Janus kinase 2 (JAK2) via its 3'-UTR region. Either inhibition of JAK2 activity by a small chemical inhibitor or downregulation of JAK2 by RNAi significantly suppressed the proliferation of gastric cancer cells. Ectopic expression of JAK2 was able to partially reverse the inhibition of cell proliferation caused by miR- 375 . Furthermore, miR-375 expression was inversely correlated with JAK2 protein level in gastric cancer. Thus, these data suggest that miR-375 may function as a tumor suppressor to influence the proliferation of gastric cancer cells by targeting the JAK2 oncogene.
A

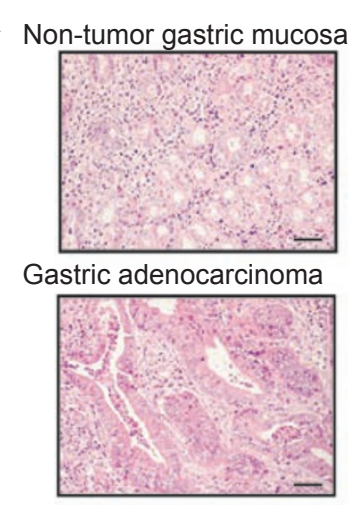

C

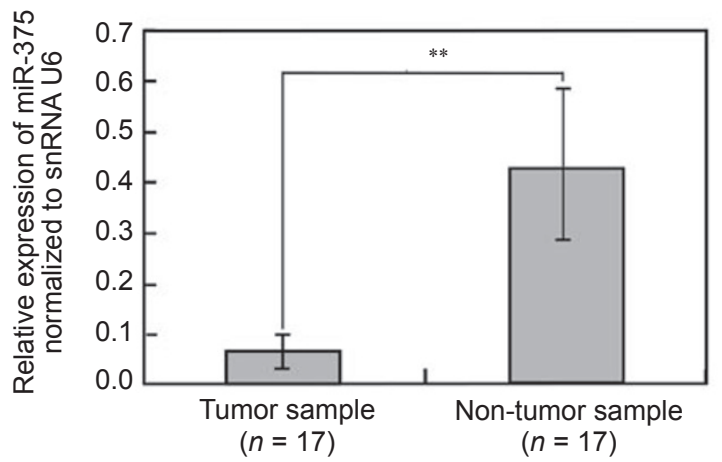

B
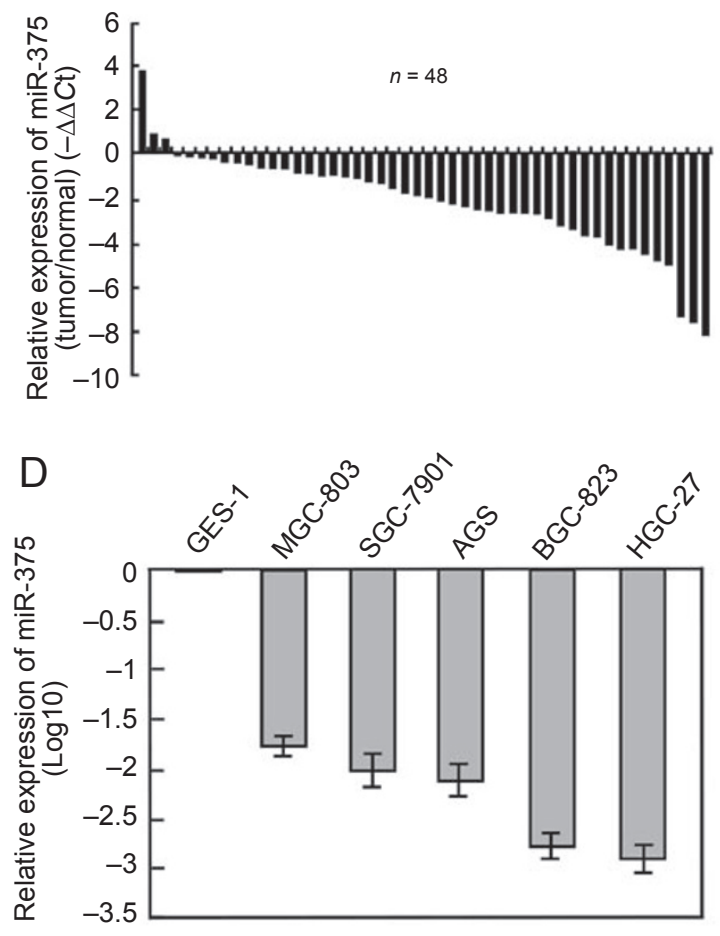

Figure 1 MiR-375 expression is frequently downregulated in human gastric cancer. (A) The miRNAs significantly associated with gastric adenocarcinoma by significance analysis of microarrays are listed. Representative histological features of nontumor gastric mucosa and gastric adenocarcinoma are shown with hematoxylin and eosin staining. Bar, $200 \mu \mathrm{m}$. (B) Relative expression of miR-375 in 48 primary gastric cancer tissues compared with their pair-matched adjacent nontumor tissues. Quantification of miR-375 was measured by qRT-PCR with specific primers for miR-375 and snRNA U6. Data are shown as $-\Delta \Delta$ Ct values. (C) MiR-375 expression was determined in age- and sex-matched endoscopic samples of gastric adenocarcinoma and nontumor mucosa. Bars represent differences in $2^{-\Delta C t}$ values between gastric adenocarcinoma and nontumor samples $(* * P<0.01)$. (D) Relative expression of miR-375 in five cell lines derived from gastric cancer and one nonmalignant gastric cell line (GES-1) was determined by qRT-PCR. Data are presented as log10 value of miR-375 in gastric cancer cell lines normalized to GES-1 cells. 
A

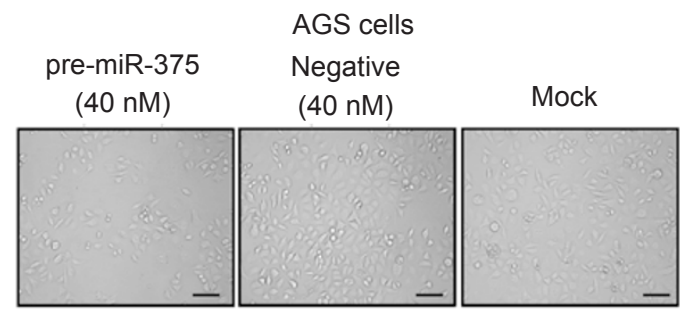

C

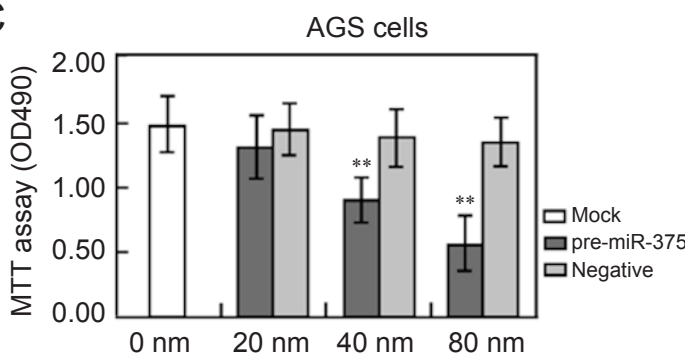

E

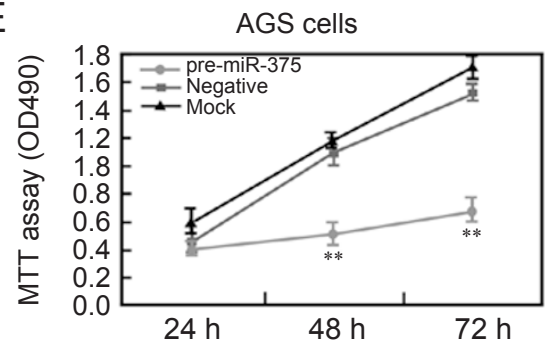

B

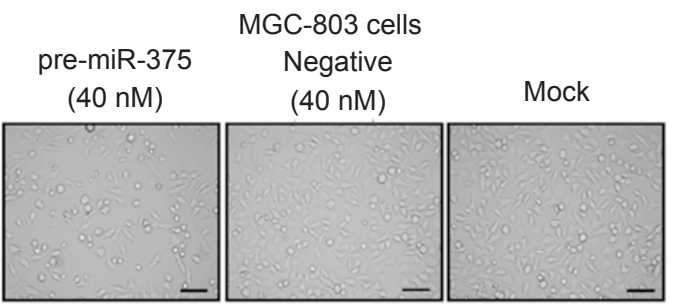

$\mathrm{D}$



$\mathrm{F}$

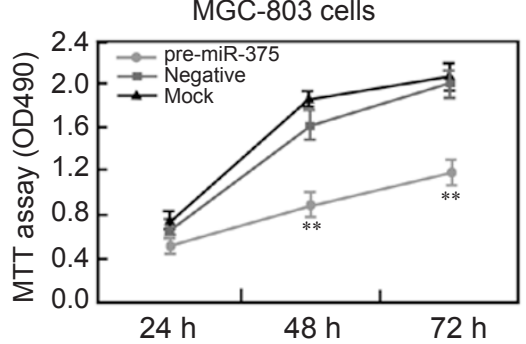

Figure 2 Effects of miR-375 on the proliferation of AGS and MGC-803 cells. The cells transfected with miR-375 precursor (pre-miR-375), negative control (negative) or neither of the above (mock) with the different concentrations for different time periods were subjected to phase-contrast microscopy and MTT analyses. (A-B) The treated cells were analyzed by phasecontrast microscopy analysis at $72 \mathrm{~h}$ post transfection. Scale bars represent $30 \mu \mathrm{m}$. (C-D) Cells transfected with different amounts of miRNA precursors for $72 \mathrm{~h}$ were subjected to MTT analysis. (E-F) The cells treated with $40 \mathrm{~nm}$ miR-375 precursor, $40 \mathrm{~nm}$ negative control or neither of the above were measured by MTT assay at different time periods. Data are shown as mean \pm SD from three independent experiments. ${ }^{*} P<0.05, * * P<0.01$ versus negative.

\section{Results}

\section{MiR-375 is frequently downregulated in gastric cancer}

To search miRNAs potentially involved in gastric tumorigenesis, we performed miRNA microarray profiling in six primary gastric tumors and their pair-matched nontumor tissues from patients undergoing gastric resection. A total of 15 miRNAs were detected to exhibit significantly differential expression in primary tumors compared to their nontumor controls by paired significance analysis of microarray (SAM) (Figure 1A). The expression pattern of four miRNAs (miR-21, miR-25, miR-106a and miR106b) was consistent with the previous profiling study, which was confirmed by other independent reports [12, 15, 17-20]. Among them, miR-375 was shown to be the most significantly downregulated miRNA in stomach tumors. Quantitative real-time RT-PCR (qRT-PCR) analysis validated that miR-375 was greatly downregulated in about $94 \%$ of tumors $(P<0.01,45$ of 48 patients), with 3.94-fold reduction relative to their adjacent non-tumor tissues (Figure 1B and Supplementary information, Table $\mathrm{S} 1)$. To further investigate the expression of miR-375 in gastric cancer, we detected the expression of miR-375 in the age- and sex-matched samples from gastroscopy in a double-blind fashion and found that miR-375 was also significantly decreased in gastric cancer mucosa compared with non-tumor mucosa $(P<0.01)$ (Figure 1C and Supplementary information, Table S2). To confirm the association between miR-375 expression and gastric cancer, we examined miR-375 expression in several gastric cancer cell lines using qRT-PCR. These data showed that miR-375 expression was substantially reduced in all the cell lines derived from gastric cancers with various differentiation degrees, compared with the nonmalignant gastric cell line GES-1 (Figure 1D) or non-tumor tissue (Supplementary information, Figure S1). Thus, these 
data suggest that miR-375 is frequently down-regulated in gastric cancer.

Overexpression of miR-375 inhibits gastric cancer cell proliferation

To explore the role of miR-375 in gastric carcinogenesis, we examined the effect of miR-375 overexpression on the proliferation of AGS and MGC-803 gastric cancer cell lines with low endogenous expression of miR-375.


Figure 3 Overexpression of miR-375 inhibits xenograft tumor formation of gastric cancer cells. MGC-803 cells with miR-375 overexpression or not were inoculated subcutaneously into both flanks of nude mice $(n=6)$. (A) Increased miR-375 expression suppresses xenograft tumor growth. The average volume of tumors developed in nude mice is shown as mean \pm SE. (B-C) The xenografts with miR-375 overexpression were significantly smaller than the control xenografts. The mice were killed and the tumors were weighted 3 weeks after inoculation. Photograph of tumors developed in each group is shown (B). Tumors from each group were weighed immediately after removal. The average tumor weight is indicated as mean \pm SE (C).
The cells were transfected with either miR-375 precursor or precursor-negative control oligonucleotides at different concentrations for various periods. The qRTPCR analysis confirmed that the expression of mature miR-375 was significantly increased in cells transfected with miR-375 precursor compared with negative control (Supplementary information, Figure S2). Phase-contrast microscopy, MTT assays and direct cell counts showed that overexpression of miR-375 significantly inhibited the proliferation of both AGS and MGC-803 cells (Figure 2 and Supplementary information, Figure S3). Collectively, these results indicate that miR-375 may play a role in the proliferation of gastric cancer cells in vitro.

Increased miR-375 expression suppresses xenograft tumor formation

To further evaluate the potential effect of miR-375 on gastric cancer cell proliferation in vivo, MGC-803 cells with or without miR-375 overexpression were subcutaneously inoculated into both flanks of athymic nude mice. The mean volume of miR-375 overexpressing tumors was significantly smaller than that of the control, 10 days after inoculation (Figure 3A). At the end of the experimental period, the mean wet weight of tumors with miR375 overexpression was significantly lower than that of the control (Figure 3B and 3C). Thus, these data indicate that miR-375 may inhibit xenograft tumor formation of gastric cancer cells in vivo.

MiR-375 represses JAK2 protein expression through 3'UTR

To investigate the mechanism of miR-375 function in gastric carcinogenesis, we employed miRanda, TargetScan and PicTar algorithms to search for putative human protein-coding gene targets of miR-375. The gene for JAK2, predicted by both TargetScan and PicTar, was further studied as a potential target (Figure 4A). Overexpression of miR-375 in AGS and MGC-803 cells greatly reduced the protein level of JAK2, whereas the level of JAK2 mRNA was not significantly affected (Figure 4B and Supplementary information, Figure S4), suggesting that miR-375 may suppress JAK2 gene expression through translational repression. To test whether the predicted miR-375-binding sites in the 3'-UTR of JAK2 mRNA were responsible for its regulation, we cloned the 3'-UTR region of JAK2 downstream of a luciferase reporter gene (pMIR-JAK2), and co-transfected this vector together with miR-375 precursor or its negative control into AGS and MGC-803 cells. The luciferase activity of cells transfected with miR-375 precursor was significantly decreased compared with the negative control $(P$ $<0.01$ ) (Figure 4C and 4D). Moreover, mutation of the 

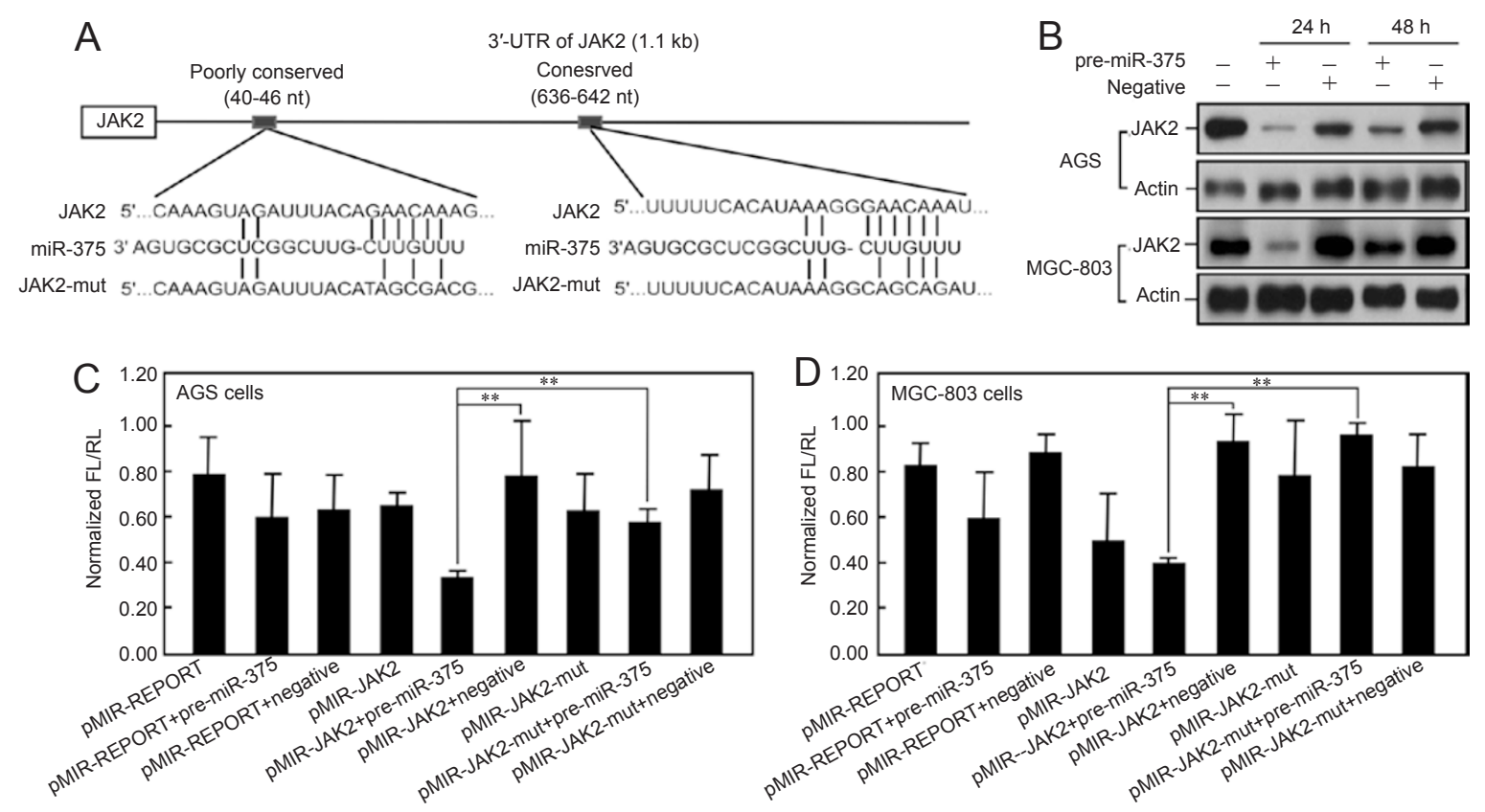

Figure 4 JAK2 is a miR-375 target. (A) Schematic of two putative miR-375-binding sites in JAK2 3'-UTR region, as detected by TargetScan. JAK2-mut indicates the JAK2 3'-UTR with mutation in miR-375-binding sites. (B) Ectopic expression of miR375 reduces JAK2 protein level in AGS and MGC-803 cells. The cells were transfected with either miR-375 precursor or negative control for different time periods and subjected to western analysis with the indicated antibodies. The level of actin was used as a loading control. (C-D) The regulation of luciferase activity by JAK2 3'-UTR is dependent on miR-375. AGS (C) and MGC-803 (D) cells were transfected with the pRL-TK containing Renilla luciferase gene and the indicated vectors or precursors. Bars indicate the Firefly luciferase activities normalized to Renilla luciferase activities of the cotransfected pRL-TK vector. Each experiment was repeated at least three times, and each sample was assayed in triplicate. ${ }^{* *} P<0.01$.

putative miR-375-binding sites clearly abrogated the repression of luciferase activity caused by miR-375 overexpression (Figure 4C and 4D). These data suggest that miR-375 may inhibit JAK2 protein expression through 3 '-UTR at the posttranscriptional level.

\section{$J A K 2$ is involved in the regulation of cell proliferation by miR-375}

Because miR-375 inhibits gastric cancer cell proliferation and suppresses JAK2 protein expression, we are interested in examining whether miR-375 plays a role in the regulation of cell proliferation via JAK2 regulation. To examine the role of JAK2 in gastric cancer cell proliferation, we used tyrphostin AG490, a JAK2-specific inhibitor [21], and found that AG490 significantly suppressed the proliferation of AGS and MGC-803 cells in a dose- and time-dependent manner (Figure 5 $\mathrm{A}$ and $5 \mathrm{~B}$ ), indicating a potential role of JAK2 in gastric cancer cell proliferation. To confirm this function of JAK2, we further employed vector-based RNAi technique to knockdown JAK2 and found that ectopic expression of pSilencer-JAK2 was able to effectively deplete JAK2, whereas the level of actin remained unchanged (Supple- mentary information, Figure S5A). Meanwhile, depletion of endogenous JAK2 inhibited the proliferation of AGS and MGC-803 cells, which was consistent with the effect of miR-375 overexpression (Figure 6). Furthermore, we examined whether JAK2 counteracted the inhibition of cell proliferation caused by miR-375 overexpression in gastric cancer cells. The vector of pCMV-JAK2 that contains only the JAK2 coding sequence was constructed for JAK2 expression without miR-375 targeting. The cells were co-transfected with miR-375 precursor and either pCMV-JAK2 or pCMV empty vector. The data clearly showed that ectopic expression of JAK2 effectively reversed the suppression of cell proliferation caused by miR-375 overexpression (Figure 6 and Supplementary information, Figure S5B). Taken together, these results are consistent with our hypothesis that miR-375 may inhibit gastric cancer cell proliferation potentially by regulating JAK2 expression.

The association of miR-375 expression with JAK2 protein levels in gastric cancer

As miR-375 was frequently down-regulated in gastric cancer and targeted JAK2 to inhibit cell proliferation, 



Figure 5 JAK2 inhibitor AG490 inhibits the proliferation of MGC-803 and AGS cells. MTT assays showed that the proliferation of AGS (A) and MGC-803 (B) cells were inhibited by AG490 in a dose- and time-dependent manner. The representative results are shown as mean \pm $\mathrm{SD}$. Each experiment was repeated at least three times, and each sample was assayed in triplicate. ${ }^{*} P<0.05$, $* * P<0.01$ versus control (AG490, $0 \mu \mathrm{M})$.

we determined whether JAK2 protein level is associated with stomach cancer. The results revealed that JAK2 protein level was significantly increased in gastric cancer samples compared with their non-tumor counterparts $(P<0.05$; Figure 7A). To assess the clinical relevance of these findings, we correlated JAK2 level with miR375 expression in the same patients. As shown in Figure $7 \mathrm{~B}$, when the relative expression levels of JAK2 (tumor/ normal) were plotted against that of miR-375 (tumor/ normal) in each patient, a significant inverse correlation was found $(P<0.05 ; r=-0.41)$. These data indicate that miR-375 down-regulation may be associated with the increase of JAK2 protein levels in gastric cancer.

\section{Discussion}

Although miRNA signatures for stomach cancer have been established, elucidation of the role of deregulated miRNAs in gastric carcinogenesis remains in the early stage of development. The data presented here showed a role of miR-375 in gastric cancer. MiR-375 is predominantly expressed in pancreatic islet, where it has been reported to regulate insulin secretion and gene expression by targeting myotrophin and 3'-phosphoinositidedependent protein kinase-1, respectively [22, 23]. MiR375 gene knockout mice revealed that miR-375 is not only required for normal glucose homeostasis but also influences pancreatic $\alpha$ - and $\beta$-cell mass [24]. Inhibition of miR-375 maturation with morpholinos in zebrafish has revealed an essential role for miR-375 in the formation of insulin-secreting pancreatic islet [25]. By far, functional studies of miR-375 appear to be restricted to the regulation of pancreas. Here, we found that miR-375 was under-expressed in more than $90 \%$ of stomach cancer samples compared with their nontumor counterparts obtained from patients undergoing gastric resection, which was confirmed by the data from gastroscopy. MiR375 was shown to inhibit gastric cancer cell proliferation partially by targeting JAK2. Moreover, miR-375 expression was inversely correlated with JAK2 protein level in gastric cancer. These findings indicate that miR-375 may function as tumor suppressor to influence gastric caner cell proliferation potentially by down-regulating JAK2 expression.

Until very recently, miR-375 has been found to be
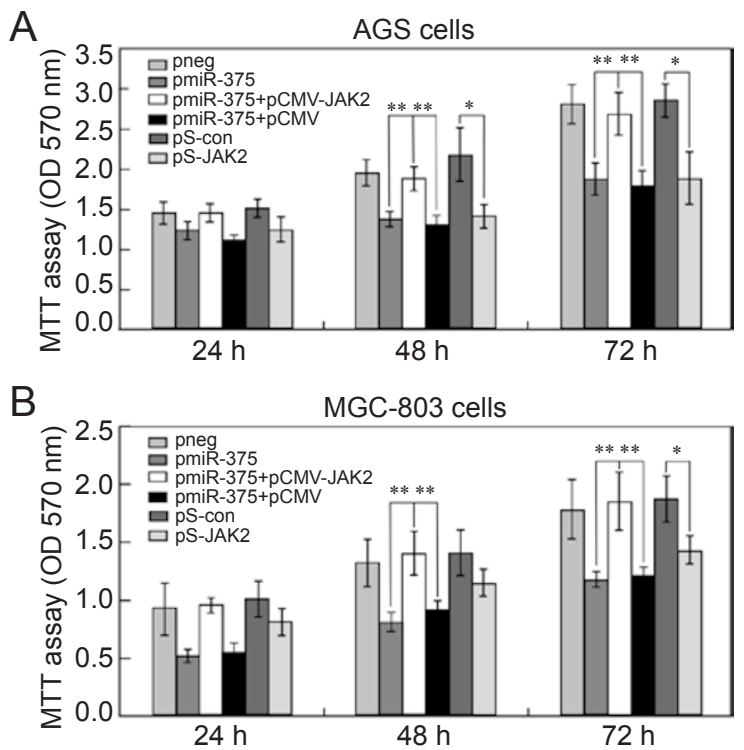

Figure 6 The role of miR-375 in the regulation of gastric cancer cell proliferation. MiR-375 overexpression suppressed AGS (A) and MGC-803 (B) cell proliferation. The cells transfected with the indicated vectors or oligonucleotides were subjected with MTT assays. The rescue experiments for miR-375 overexpression were performed by ectopic expression of JAK2 without 3'-UTR in miR-375-treated cells. All the experiments were performed at least three times and a representative result is displayed as mean \pm SD. $* P<0.05, * * P<0.01$. 

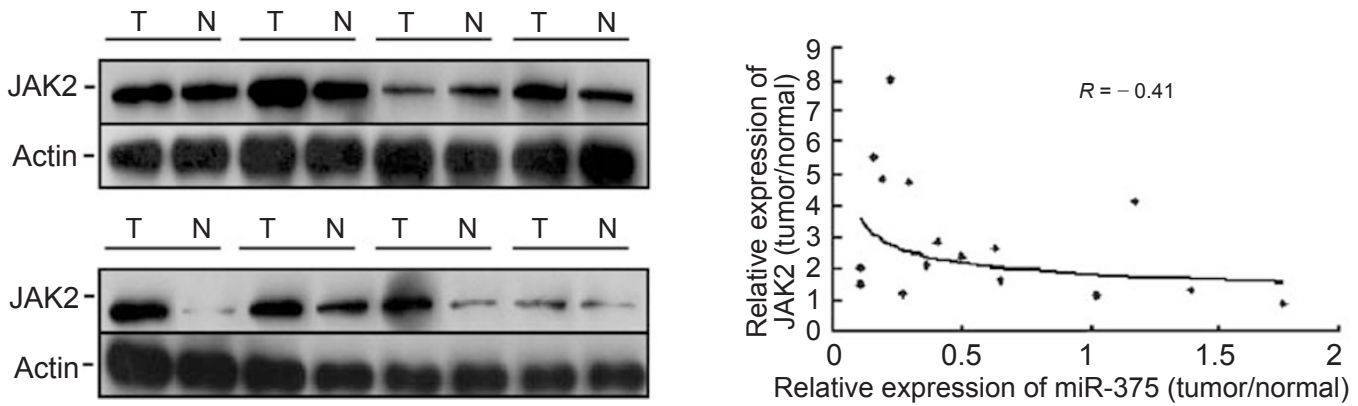

Figure 7 JAK2 protein and miR-375 expression correlates in gastric cancer. (A) JAK2 is significantly upregulated in primary gastric cancers compared to their paired nontumor gastric tissues. The tissue samples were subjected to western blot analysis with the indicated antibodies. Actin was used as a loading control. (B) Inverse correlation between miR-375 expression and JAK2 protein in primary gastric cancers versus their pair-matched nontumor counterparts. A statistically significant correlation between miR-375 expression and JAK2 protein was observed by Pearson's method with a correlation coefficient of -0.41 .

deregulated in some malignancies, including live tumor with $\beta$-catenin mutation, head and neck squamous cell carcinoma and pancreatic adenocarcinomas [26-29], whereas little is known about the molecular targets of miR-375 in these cancer. To our knowledge, JAK2 may be the first identified target gene that is involved in the regulation of carcinogenesis by miR-375.

The Janus kinase/signal transducer and activator of transcription (JAK/STAT) pathway mediates signaling by many cytokines, which is required for cell proliferation, survival and differentiation [30]. JAK2 is a member of the Janus family of cytoplasmic non-receptor tyrosine kinases, which also includes JAK1, JAK3 and TYK2 [31]. The V617F mutation of JAK2 is detected in many patients with polycythemia vera, essential thrombocythemia and primary myelofibrosis, resulting in activation of its tyrosine kinase activity, phosphorylation of STATs and expression of target genes [32-35]. However, little is known about the role of JAK2 in solid tumors, especially in gastric cancer. A recent report failed to detect JAK2 mutation in gastric cancer [36]. Here, for the first time, we provide evidence that upregulation of JAK2 is associated with gastric cancer. Furthermore, down-regulation of JAK2 by either miR-375 overexpression or RNAi and inhibition of JAK2 activity by small molecule inhibitor were found to significantly suppress the proliferation of gastric cancer cells. These data suggest that JAK2 may play a role in gastric carcinogenesis.

Targeting JAK2 has emerged as an attractive strategy of novel drug development because it is mutated in many patients with myeloproliferative disorders [31, 37]. Most of the JAK2-directed drug discovery efforts are in early/ mid-stage of clinical development or are yet to reach the clinic. Of these small molecule inhibitors, lestaurtinib and INCB-18424 are currently being evaluated in phase II clinical trials [37]. Thus, it will be very interesting to evaluate the effectiveness of these molecules in the treatment of gastric cancer.

Additionally, identification of miR-375 downregulation in most gastric cancers may provide a new therapeutic opportunity. Studies regarding therapeutic utilization of miRNAs yielded encouraging results. The development of modified miRNA molecules with longer in vivo half-life and higher efficiency, such as the locked nucleic acid-modified oligonucleotides and the antisense oligonucleotides termed 'antagomirs', has produced favorable antitumor outcomes in experimental models [36, 38]. Enforced expression of miR-375 in gastric cancer by transfection of synthetic miR-375 oligonucleotides, infection of miR-375-carrying viruses or other approaches to increase endogenous miR-375, will be clearly needed for further studies.

\section{Materials and Methods}

\section{Samples}

Pair-matched tumorous and adjacent nontumorous gastric tissues from 48 patients undergoing resection for gastric cancer were obtained from Sir Run Run Shaw Hospital, Zhejiang University School of Medicine (Hangzhou, China). Written informed consent was obtained before collection. Non-tumor samples from the macroscopic tumor margin were isolated at the same time and used as the matched adjacent non-neoplastic tissues. All the samples were divided into two parts. One part was immediately snap frozen and stored in liquid nitrogen until RNA extraction. Another part was stored in formalin for pathology analysis. The histopathological investigations including hematoxylin and eosin staining were performed by two professional pathologists independently. Other clinical data were obtained from medical records within Sir Run Run Shaw Hospital, China. The clinicopathological characteristics 
of included patients are summarized in Supplementary information, Table S1. Gastric mucosa samples including 17 nonmalignant mucosa and 17 malignant mucosa that were obtained from gastroscopy were also stored in liquid nitrogen until use. The clinical characteristics of these patients are reviewed in Supplementary information, Table S2.

\section{$R N A$ extraction}

Total RNA from tissue samples and cell lines was extracted using the mirVana miRNA Isolation Kit (Ambion, TX, USA) following the manufacture's protocol. RNA concentrations and quality were determined with a NanoDrop ND-1000 spectrophotometer (NanoDrop Technologies, DE, USA) and gel analysis.

\section{miRNA microarray}

Microarray analysis was performed on $5 \mu \mathrm{g}$ of total RNA from histologically confirmed gastric cancers and adjacent normal tissues from the same patient, and was carried out using the $\mu$ Paraflo microfluidic technology according to the manufacturer's protocol (LC Sciences, TX, USA). Hybridization was performed overnight on a microfluidic chip. On the microfluidic chip, each detection probe consisted of a chemically modified nucleotide-coding segment complementary to target miRNA and a spacer segment of polyethylene glycol to extend the coding segment away from the substrate. The array covers all miRNA transcripts available in the Sanger miRBase database (release 10). Post-hybridization, detection used fluorescence labeling with tag-specific $\mathrm{Cy} 3$ and Cy5 dyes. Hybridization images were collected using a GenePix 4000B laser scanner (Molecular Device, CA, USA) and digitized using Array-Pro image analysis software (Media Cybernetics, MD, USA). The data were analyzed by first subtracting the background and then normalizing the signals to balance the intensities of the Cy3 and Cy5-labeled transcripts so that differential expression ratios can be calculated. The ratio of the two sets of detected signals $\left(\log _{2}(\right.$ cancer/normal $\left.)\right)$ and $P$-values of the $t$-test were calculated; differentially detected signals were those with a $P$-value less than 0.05 . Deregulated miRNAs were divided into high or low signal using a signal intensity value of 500 normalized signal units.

\section{Construction of plasmids}

To produce pMIR-JAK2 plasmid, human JAK2 3'-UTR amplified by RT-PCR using the primers $5^{\prime}$-AAG AAA TGA CCT TCA TTC TGA GA-3' and 5'-GTT ATG AAT TTT CTA CTG GCA TTC-3' was cloned into pMIR-REPORT vector (Ambion). The mutation on miR-375-binding sites in human JAK2 3'-UTR was also generated by overlap PCR, as previously described [39]. For ectopic expression of FLAG-tagged JAK2, human JAK2 with coding region was cloned into pCMV-Tag $2 \mathrm{C}$ vector. To deplete endogenous JAK 2 by RNAi, the oligo containing complementary hairpin sequences were synthesized and cloned into pSilencer 4.1CMV neo vector (Ambion). The targeting sequence of JAK2 gene is 5'-AAC TCT ATC AGC TAC AAG ACA-3', corresponding to the region 604-624 relative to the first nucleotide of the start codon. A circular pSilencer 4.1-CMV neo vector that expresses a hairpin siRNA with limited homology to any known sequence was used as a negative control. To construct the plasmid that expresses miR-375 in mammalian cells, the paired oligonucleotides based on the primary sequence of has-miR-375 and its flanking regions were cloned into mammalian expression vector pEGFP-C1 (Clontech,
CA, USA). All of these constructs were confirmed by sequencing.

\section{Cell culture}

The biological characteristics and source of gastric cancer cell lines were summarized in our previous report [9]. The nonmalignant gastric cell line GES-1 was purchased from the Beijing Institute for Cancer Research (Beijing, China). These cells were maintained at $37{ }^{\circ} \mathrm{C}$ in an atmosphere of $5 \% \mathrm{CO}_{2}$ in RPMI-1640 (MGC-803, BGC-823, SGC-7901), DMEM (GES-1, HGC-27) or F12 (AGS) medium supplemented with $10 \%$ fetal bovine serum, penicillin and streptomycin (Gibco BRL, NY, USA) in 25-ml culture flasks.

\section{Real-time PCR analysis}

The expression of mature miRNAs was assayed using the Taqman MicroRNA Assays (Applied Biosystems, CA, USA) with specific primers for hsa-miR-375 (P/N: 4373151, Applied Biosystems) on tissue samples and cell lines. Reverse transcription reaction was carried out starting from $10 \mathrm{ng}$ of total RNA using the looped primers. Real-time PCR was performed using the standard Taqman MicroRNA assays protocol on ABI7500 real-time PCR detection system. The $\Delta \Delta C \mathrm{t}$ method for relative quantization was used to determine miRNA expression. The $C t$ is the fractional cycle number at which the fluorescence of each sample passes the fixed threshold. The $\Delta C \mathrm{t}$ was calculated by subtracting the $C \mathrm{t}$ of snRNA U6 (RNU6B, P/N: 4373381, Applied Biosystems) from the $C \mathrm{t}$ of the miRNA of interest. The $\Delta \Delta C \mathrm{t}$ was calculated by subtracting the $\Delta C \mathrm{t}$ of the reference sample (paired nontumorous tissue for surgical samples, normal tissues and GES-1 cell for five gastric cancer cell lines) from the $\Delta C t$ of each sample. Fold change was determined as $2^{-\Delta \Delta C t}$ and miR-375 expression in endoscopic samples was also normalized to snRNA U6 using the $2^{-\Delta C t}$ method.

\section{Cell proliferation assay}

To determine the effect of miR-375 on proliferation of gastric cancer cell lines, $4 \times 10^{3}$ cells were transfected with Pre-miR miR375 precursor molecule (miR-375 precursor), Pre-miR miRNA precursor molecules' negative control no. 1 (negative control) using siPORT amine transfection agent (Ambion) or with mock control following manufacturer's protocol in 96-well plate. MTT (3-(4, 5-dimethylthiazol-2-yl)-2, 5-diphenyl-tetrazoliumbromide) assay was performed at $24 \mathrm{~h}, 48 \mathrm{~h}$ and $72 \mathrm{~h}$ post transfection. The absorbance of the samples was measured with a spectrophotometer reader at $490 \mathrm{~nm}$. Each assay was performed in triplicate and repeated three times independently.

\section{Xenograft tumor model}

Animal experiments were conducted in accordance with the Guide for the Care and Use of Animals for research purposes, and were approved by the Committee of Animal Ethics, Zhejiang University. Female athymic nude (nu/nu) mice (3-4 weeks old) were purchased from Shanghai Laboratory Animal Co Ltd (SLAC, Shanghai, China). The mice were bred and maintained under specific pathogen-free conditions in the Animal Facility, Zhejiang University. MGC-803 cells $\left(5 \times 10^{6}\right)$ transfected with either the vector overexpressing miR-375 or the control vector were inoculated subcutaneously into the left and right flanks of nude mice, respectively. The volume of the implanted tumor was measured at every 2 days with a vernier caliper, using the formula: $V=L \times W^{2} / 2$; 
where $V$, volume $\left(\mathrm{mm}^{3}\right) ; L$, biggest diameter $(\mathrm{mm}) ; W$, smallest diameter $(\mathrm{mm})$. The mice were killed and the tumors were weighd 3 weeks after inoculation.

\section{Luciferase activity assay}

In total, 40000 cells were seeded in 24 -well plates $24 \mathrm{~h}$ prior to transfection. Cells were cotransfected with $0.1 \mu \mathrm{g}$ of either pMIRJAK2 or pMIR-REPORT together with $40 \mathrm{nM}$ of miR-375 precursor molecule or $40 \mathrm{nM}$ of negative control no. 1 using siPORT amine transfection agent (Ambion) following manufacturer's protocol. The pRL-TK vector (Promega, WI, USA) containing Renilla luciferase was also cotransfected as a reference control. Firefly and Renilla luciferase activities were measured by using dualluciferase reporter assay (Promega) $24 \mathrm{~h}$ after transfection. Firefly luciferase activity was normalized to Renilla luciferase activity.

\section{Western blotting}

Western blotting analyses were performed as described previously [40]. In brief, the cells transfected with the indicated vectors or chemicals were subjected to western blotting with anti-JAK2 (Cell signaling, MA, USA) and actin (Santa Cruz Biotechnology, CA, USA) antibodies, respectively. Actin was used as a loading control.

\section{Statistical analysis}

Differential expressions of miRNA in surgical samples were determined by testing the null hypothesis that the mean of log2transformed values of fold change was equal to 0 by one-sample $t$-test. Student's $t$-test was applied to determine the differences between sample means obtained from at least three experiments. Relationships between miR-375 and JAK2 expression were explored by Pearson's correlation coefficient, which is a technique for measuring the strength of the association between two quantitative, continuous variables. The Pearson's correlation coeffcient $(R)$ may take any value from -1 to +1 . The nearer the absolute value of $R$ is to 1 , the higher the strength of association between the variables. $P<0.05$ was considered to be statistically significant. Statistical analyses were performed with SPSS software (version 13).

\section{Acknowledgments}

This work was supported by the National Natural Scientific Foundation of China (30901714, 30671070 and 30771107), the Ministry of Science and Technology of China (2007CB914500), the Ministry of Education of China (NCET-06-0530), the Ministry of Health of China (WKJ2006-2-014), the Postdoctoral Science Foundation of China (20070421179), the Department of Science and Technology of Zhejiang Province (2009F80032), and the Natural Scientific Foundation of Zhejiang Province, China (R205291, Y206103 and 2007R10G2010103).

\section{References}

1 Ambros V. The functions of animal microRNAs. Nature 2004; 431:350-355.

2 Bartel DP. MicroRNAs: genomics, biogenesis, mechanism, and function. Cell 2004; 116:281-297.

3 Pasquinelli AE, Hunter S, Bracht J. MicroRNAs: a developing story. Curr Opin Genet Dev 2005; 15:200-205.
4 Ahmed FE. Role of miRNA in carcinogenesis and biomarker selection: a methodological view. Expert Rev Mol Diagn 2007; 7:569-603.

5 Calin GA, Sevignani C, Dumitru CD, et al. Human microRNA genes are frequently located at fragile sites and genomic regions involved in cancers. Proc Natl Acad Sci USA 2004; 101:2999-3004.

6 Croce CM. Causes and consequences of microRNA dysregulation in cancer. Nat Rev Genet 2009; 10:704-714.

7 Parkin DM, Bray F, Ferlay J, Pisani P. Global cancer statistics, 2002. CA Cancer J Clin 2005; 55:74-108.

8 Hohenberger P, Gretschel S. Gastric cancer. Lancet 2003; 362:305-315.

9 Yasui W, Yokozaki H, Fujimoto J, et al. Genetic and epigenetic alterations in multistep carcinogenesis of the stomach. $J$ Gastroenterol 2000; 35 Suppl 12:111-115.

10 Stock M, Otto F. Gene deregulation in gastric cancer. Gene 2005; 360:1-19.

$11 \mathrm{Du} \mathrm{Y}, \mathrm{Xu} \mathrm{Y}$, Ding L, et al. Down-regulation of miR-141 in gastric cancer and its involvement in cell growth. $J$ Gastroenterol 2009; 44:556-561.

12 Takagi T, Iio A, Nakagawa Y, et al. Decreased expression of microRNA-143 and -145 in human gastric cancers. Oncology 2009; 77:12-21.

13 Luo H, Zhang H, Zhang Z, et al. Down-regulated miR-9 and miR-433 in human gastric carcinoma. J Exp Clin Cancer Res 2009; 28:82.

14 Gao C, Zhang Z, Liu W, et al. Reduced microRNA-218 expression is associated with high nuclear factor kappa B activation in gastric cancer. Cancer; 116:41-49.

15 Chan SH, Wu CW, Li AF, Chi CW, Lin WC. miR-21 microRNA expression in human gastric carcinomas and its clinical association. Anticancer Res 2008; 28:907-911.

$16 \mathrm{Wu} \mathrm{Q}$, Jin $\mathrm{H}$, Yang Z, et al. MiR-150 promotes gastric cancer proliferation by negatively regulating the pro-apoptotic gene EGR2. Biochem Biophys Res Commun 2010; 392:340-345.

17 Xiao B, Guo J, Miao Y, et al. Detection of miR-106a in gastric carcinoma and its clinical significance. Clin Chim Acta 2009; 400:97-102.

18 Zhang Z, Li Z, Gao C, et al. miR-21 plays a pivotal role in gastric cancer pathogenesis and progression. Lab Invest 2008; 88:1358-1366.

19 Petrocca F, Visone R, Onelli MR, et al. E2F1-regulated microRNAs impair TGFbeta-dependent cell-cycle arrest and apoptosis in gastric cancer. Cancer Cell 2008; 13:272-286.

20 Kim YJ, Hwang SJ, Bae YC, Jung JS. MiR-21 regulates adipogenic differentiation through the modulation of TGF-beta signaling in mesenchymal stem cells derived from human adipose tissue. Stem Cells 2009; 27:3093-3102.

21 Meydan N, Grunberger T, Dadi H, et al. Inhibition of acute lymphoblastic leukaemia by a Jak-2 inhibitor. Nature 1996; 379:645-648.

22 Poy MN, Eliasson L, Krutzfeldt J, et al. A pancreatic isletspecific microRNA regulates insulin secretion. Nature 2004; 432:226-230.

23 El Ouaamari A, Baroukh N, Martens GA, et al. miR-375 targets 3'-phosphoinositide-dependent protein kinase-1 and regulates glucose-induced biological responses in pancreatic beta-cells. Diabetes 2008; 57:2708-2717. 
24 Poy MN, Hausser J, Trajkovski M, et al. miR-375 maintains normal pancreatic alpha- and beta-cell mass. Proc Natl Acad Sci USA 2009; 106:5813-5818.

25 Kloosterman WP, Lagendijk AK, Ketting RF, Moulton JD, Plasterk RH. Targeted inhibition of miRNA maturation with morpholinos reveals a role for miR-375 in pancreatic islet development. PLoS Biol 2007; 5:e203.

26 Ladeiro Y, Couchy G, Balabaud C, et al. MicroRNA profiling in hepatocellular tumors is associated with clinical features and oncogene/tumor suppressor gene mutations. Hepatology 2008; 47:1955-1963.

27 Avissar M, Christensen BC, Kelsey KT, Marsit CJ. MicroRNA expression ratio is predictive of head and neck squamous cell carcinoma. Clin Cancer Res 2009; 15:2850-2855.

28 Bloomston M, Frankel WL, Petrocca F, et al. MicroRNA expression patterns to differentiate pancreatic adenocarcinoma from normal pancreas and chronic pancreatitis. JAMA 2007; 297:1901-1908.

29 Mathe EA, Nguyen GH, Bowman ED, et al. MicroRNA expression in squamous cell carcinoma and adenocarcinoma of the esophagus: associations with survival. Clin Cancer Res 2009; 15:6192-6200.

30 Constantinescu SN, Girardot M, Pecquet C. Mining for JAKSTAT mutations in cancer. Trends Biochem Sci 2008; 33:122131.

31 Levine RL, Pardanani A, Tefferi A, Gilliland DG. Role of JAK2 in the pathogenesis and therapy of myeloproliferative disorders. Nat Rev Cancer 2007; 7:673-683.
32 Levine RL, Wadleigh M, Cools J, et al. Activating mutation in the tyrosine kinase JAK2 in polycythemia vera, essential thrombocythemia, and myeloid metaplasia with myelofibrosis. Cancer Cell 2005; 7:387-397.

33 James C, Ugo V, Le Couedic JP, et al. A unique clonal JAK2 mutation leading to constitutive signalling causes polycythaemia vera. Nature 2005; 434:1144-1148.

34 Baxter EJ, Scott LM, Campbell PJ, et al. Acquired mutation of the tyrosine kinase JAK2 in human myeloproliferative disorders. Lancet 2005; 365:1054-1061.

35 Kralovics R, Passamonti F, Buser AS, et al. A gain-offunction mutation of JAK2 in myeloproliferative disorders. $N$ Engl J Med 2005; 352:1779-1790.

36 Lee JW, Soung YH, Kim SY, et al. Absence of JAK2 V617F mutation in gastric cancers. Acta Oncol 2006; 45:222-223.

37 Kumar C, Purandare AV, Lee FY, Lorenzi MV. Kinase drug discovery approaches in chronic myeloproliferative disorders. Oncogene 2009; 28:2305-2313.

38 Orom UA, Kauppinen S, Lund AH. LNA-modified oligonucleotides mediate specific inhibition of microRNA function. Gene 2006; 372:137-141.

39 Zhou T, Zimmerman W, Liu X, Erikson RL. A mammalian NudC-like protein essential for dynein stability and cell viability. Proc Natl Acad Sci USA 2006; 103:9039-9044.

40 Cai Y, Yang Y, Shen M, Zhou T. Inhibition of cytokinesis by overexpression of NudCL that is localized to the centrosome and midbody. Cell Res 2009; 19:1305-1308.

(Supplementary information is linked to the online version of the paper on Cell Research website.) 